\title{
Stroke Incidence and Death in Atrial Fibrillation Patients Newly Treated with Direct Oral Anticoagulants
}

This article was published in the following Dove Press journal: Clinical Epidemiology

\author{
Amélie Gabet ${ }^{\prime}$ \\ Edouard Chatignoux' \\ Clémence Grave \\ Alexandre Vallée ${ }^{2}$ \\ Philippe Tuppin (10 ${ }^{3}$ \\ Yannick Béjot $\mathbb{D}^{4}$ \\ Valérie Olié ${ }^{\prime}$ \\ 'French Public Health Agency, Saint- \\ Maurice, France; ${ }^{2}$ Diagnosis and \\ Therapeutic Center, Hotel Dieu, AP-HP, \\ University Paris Descartes, Paris, France; \\ ${ }^{3}$ General Health Insurance Scheme \\ (Caisse Nationale d'Assurance Maladie), \\ Paris, France; ${ }^{4}$ Dijon Stroke Registry, \\ EA4I84, University Hospital and Medical \\ School of Dijon, University of Burgundy, \\ Dijon, France
}

Correspondence: Amélie Gabet French Public Health Agency, 14 rue du Val d'Osne, Saint Maurice, 94410 , France Tel +33155125164

Fax +33155125403

Email Amelie.

GABET@santepubliquefrance.fr
Purpose: The objectives were to analyse the determinants of stroke incidence and mortality as a competing event in $\mathrm{AF}$ patients newly treated with DOAC and to assess the impact of non-adherence to DOAC treatment.

Methods: It is a population-based retrospective cohort study using the French national healthcare data system. AF patients aged $>20$ years were affiliated to the general health insurance scheme (66\% of the French population) and newly treated with DOAC between 2012 and 2015 were included and followed for 2 years.

Results: Overall 76,795 AF patients were newly treated with DOAC in 2015. Stroke incidence reached 10.1 (95\% CI: 9.6-10.6) per 1000 person-year and death 39.7 (95\% CI: 38.6-40.8) as a competitive risk. Female sex was associated with a lower risk of death but not of stroke. Non-adherence to DOAC treatment increased the risk of both stroke $(42 \%)$ and death $(38 \%)$. Acute coronary syndrome was associated with an increased risk of stroke alone, whereas heart failure decompensation, social deprivation, and haemorrhage were associated with an increased risk of death alone.

Conclusion: Both stroke and death risks remain non-negligible in AF patients treated with DOAC. Non-adherence was associated with an increased risk of stroke and death.

Keywords: atrial fibrillation, stroke, death, adherence, direct oral anticoagulants

\section{Introduction}

Atrial fibrillation (AF) has become a major public health concern with increasing incidence and prevalence due to ageing population, the increased prevalence of several vascular risk factors, and a better survival from cardiovascular disease in younger individuals. AF is also associated with a high risk of stroke and death. The majority of AF patients are now treated with oral anticoagulants (OAC) to prevent stroke or systemic embolism; according to data from the GARFIELD-AF, 71.1\% were treated as such in 2014-2015. ${ }^{1}$ Determinants of stroke in AF patients treated with OAC are rarely described, although they are well established in AF patients not using anticoagulants with several risk stratification scores. These scores are highly useful to identify patients who would otherwise benefit from anticoagulation therapy, but they are not used to detect stroke risk after anticoagulation initiation. Therefore, a better understanding of stroke incidence in AF patients treated with $\mathrm{OAC}$ is needed. In particular, the effectiveness of OAC treatment still depends on treatment adherence, even if OAC efficacy has been demonstrated in several trials. ${ }^{2}$ Several studies have pointed out the low adherence to OAC treatment, and submit your manuscript DovePress

http://c 
a decrease in adherence over time. ${ }^{2}$ This observation was made for both vitamin $\mathrm{K}$ antagonists (VKA) and direct oral anticoagulants (DOAC). ${ }^{3}$ A recent meta-analysis estimated an only $66 \%$ good adherence to DOACs in AF patients and pointed out the increased risk of thromboembolism in non-adherent patients. ${ }^{3}$ The better adherence or persistence to DOAC compared to VKA is still being discussed, as studies have yet to find any differences, ${ }^{4}$ including in France. ${ }^{5}$ Furthermore, several studies have shown that non-adherence to VKA therapy was associated with an increased risk of stroke, ${ }^{6}$ similarly to DOAC. ${ }^{3,7}$ Nevertheless, very few studies have reported the association between non-adherence to DOAC and the incidence of thrombo-embolic events, and almost none after more than 1-year of follow-up.

Limited real-life epidemiological data have evaluated the impact of OAC treatment adherence as well as the determinants of stroke incidence in $\mathrm{AF}$ patients treated with OAC. The objectives of this study were therefore to estimate the impact of non-adherence to DOAC treatment on stroke incidence and mortality as competing events in AF patients newly treated with DOAC and to identify the determinants of these outcomes.

\section{Methods}

\section{Data Source}

We used data from the French national healthcare data system (Système national des données de santé - SNDS) to identify patients newly treated with DOAC for AF indication. ${ }^{8}$ The SNDS comprehensively collects individual outpatient data, as well as healthcare prescriptions and procedures reimbursed by the French national health insurance, but it does not provide any clinical data concerning the results of medical visits, prescriptions, or examinations. ${ }^{8}$ Nevertheless, it includes information on the presence of long-term chronic diseases (LTD) eligible for $100 \%$ reimbursement of healthcare expenditure after a request by the patient's general practitioner. All this information is linked to the national hospital discharge database including data concerning public and private hospital stays (short-stay hospitals and rehabilitation services) ${ }^{8}$

\section{Study Population}

AF patients newly treated with DOAC in France were selected using an algorithm described elsewhere and using both in-patient and out-patient data. ${ }^{9}$ We restricted our analyses to patients aged over 20 years, residing in metropolitan France (ie, not in the French overseas territories of Reunion Island, French Guiana, Martinique, Guadeloupe, and Mayotte). Finally, the study population was restricted to patients affiliated to the general health insurance scheme (ie, $66 \%$ of the French population), as the date of death could not be exhaustively reported for the other insurance schemes.

\section{Follow-Up and Outcomes}

The inclusion date corresponded to the date following the period covered by the first DOAC reimbursement. Patients were followed for two calendar years or until the occurrence of one of the following events: death (based on vital status), hospitalization for stroke (ICD-10 codes I60-I64 as primary diagnosis), or another censoring event such as switch to VKA, any hospitalization for systemic embolism (SE), transient ischemic attack, or haemorrhage (see Supplementary Table I for the ICD-10 codes). The main outcome was the first hospitalization for stroke using death as a competing event. In sensitivity analyses, we also studied ischemic stroke and all strokes/SE as main outcomes (see Supplementary Table II). Secondary analyses with Major Adverse Cardiovascular Events (MACE), including all-cause death, stroke, systemic embolism and myocardial infarction (MI) as outcomes were also performed (see Supplementary Table II).

At the time of inclusion, we recorded the following data: age; sex; hospitalization or long-term disease status (LTD) within the 2 previous calendar years for stroke, heart failure, acute coronary syndrome, haemorrhage, or acute kidney disease; and medication reimbursement for antihypertensive drugs, antidiabetics, lipid-lowering drugs, and oral antiplatelet agents in the previous years (if at least 3 reimbursements in the year preceding inclusion). Furthermore, each patient included in the study was attributed a social deprivation index score according to their area of residence, an index developed. ${ }^{10} \mathrm{CHA}_{2} \mathrm{DS}_{2}$-VASc score was computed for complementary analyses (see Supplementary Table III).

\section{Adherence Definition}

Adherence was defined as the number of days covered by the DOAC treatment. We recorded all DOAC reimbursements until the end of follow-up. The number of days covered by the DOAC treatment was calculated by the number of boxes dispensed multiplied by the number of pills per box and divided by the number of pills to be taken daily according to DOAC treatment. The dosage of the first DOAC reimbursement was also recorded. If the 
patients were hospitalized during follow-up, including in rehabilitation services, the number of days spent in hospital was considered to cover the DOAC treatment with inhospital pills. Finally, the number of days covered did not go past the end of the follow-up. Patients were considered adherent if the number of DOAC pills dispensed covered more than $80 \%$ of the follow-up days, or in other words, the percentage of days covered (PDC) $>80 \%$ as used in many previous studies. ${ }^{3}$ Sensitivity analyses were performed using higher PDC thresholds.

\section{Statistical Analysis}

We calculated the crude incidence rates for stroke and death when it preceded stroke. Kaplan-Meier curves were computed. We studied the association between several patients' characteristics and time to stroke. For this purpose, we used Fine \& Gray regression models with stroke as main outcome and taking into account death without stroke as a competing event. ${ }^{11}$ We calculated subdistribution hazard ratio (SHR) for stroke. SHR can be interpreted as the risk of a factor on the incidence of stroke and death without stroke. Then, we specified cause-specific Cox regression models to compute cause-specific hazard ratios (CHR) for stroke and death, respectively. In these models, the estimation of the hazard function is computed for one outcome only, the other competing cause being censored. Reporting both SHR for stroke, CHR for stroke and CHR for death allowed to better understand the effect of each covariate on stroke risk. For both Fine \& Gray and Cox models, proportional hazard assumptions were verified.

Multivariate analyses took into account: age, sex, social deprivation index of the city of residence, year of DOAC initiation, adherence to DOAC, dosage of the first DOAC delivered, source of AF identification, AF-related medical procedures, comorbidities in the 2 years preceding inclusion (stroke, heart failure, acute coronary syndrome, haemorrhage, acute kidney disease), treatments of the vascular risk (antihypertensive, lipid-lowering, antidiabetic and antiplatelet drugs).

SAS (version 7.11, SAS Institute Inc., Cary, NC, USA) and $\mathrm{R}$ software (version 3.4.3.) were used for statistical analyses.

\section{Results}

In 2015, 76,795 patients were included in the study. Median age was 75 years, with $62 \%$ of patients aged between 65 and 84 years (Table 1). Women represented $46.0 \%$ of patients, and they had a higher median age than men (78 vs 72 years). Overall, $21.0 \%$ of patients were in the highest quintile of social deprivation compared to $19.1 \%$ in the lowest quintile. History of stroke was reported in $6.4 \%$ of patients and history of heart failure decompensation in $12.9 \%$ of patients. Treatments for hypertension, diabetes, and hyperlipidaemia were observed in $73.4 \%, 18.9 \%$, and $40.5 \%$ of patients, respectively. Women, who were generally older, had higher rates of history of stroke, heart failure, and antihypertensive treatment compared to men, but they were less likely to have acute coronary syndrome and diabetes or take lipidlowering drugs.

Adherence to DOAC treatment reached $66.6 \%$ of patients in the 2015 cohort during the follow-up period. Adherent patients were older, more socially deprived, and had more history of stroke $(7.9 \%$ vs $3.4 \%)$ and heart failure $(15.3 \%$ vs $12.2 \%)$ compared to non-adherent patients (Table 1). They also were more frequently treated for arterial hypertension (78.3\% vs $64.1 \%)$, diabetes $(20.8 \%$ vs $15.6 \%$ ), and hyperlipidaemia (45.0\% vs $31.8 \%)$. Adherence was significantly higher in women $(68.9 \%)$ than in men (64.7\%). Furthermore, women were less likely to receive standard DOAC doses $(51.3 \%$ vs $67.1 \%$ in men).

Over a median follow-up of 700 days for the 2015 cohort, the incidence of stroke was 10.1 (95\% CI: 9.6-10.6) per 1000 person-year, being 7.2 (95\% CI: 6.8-7.7) for ischemic stroke and 13.2 (95\% CI: 12.6-13.9) for all strokes/SE. The incidence of death before stroke was 39.7 (95\% CI: 38.6-40.8) per 1000 person-year in 2015. Crude incidences of stroke and death, respectively, were lower in adherent patients compared to non-adherent patients, as shown in the Kaplan-Meier curves in Figure 1 (A (stroke or death), B (stroke), C (death)) and Table 1.

Results from multivariate analyses are shown in Table 2. Non-adherence to DOAC treatment was associated with a substantial increase in the risk of stroke (SHR 1.42, 95\% CI: 1.33-1.51) and death (CHR 1.38, 95\% CI: 1.33-1.43) after adjustments. Patients receiving a low dose of OAC at initiation were also at a higher risk of stroke (SHR 1.10, 95\% CI: 1.02-1.18) and death (CHR 1.31, 95\% CI: 1.26-1.36) compared to those initiating OAC at the standard dose. Stroke and death risks increased in AF patients who were identified by hospitalization with AF, an AF-specific medical procedure, or an AF long-term disease status compared to outpatients, as well as in patients with a history of stroke in the 2 previous years (SHR 2.60, 95\% CI: 2.38-2.84 and CHR 1.10, 95\% CI: 


\begin{tabular}{|c|c|c|c|c|c|c|c|c|}
\hline 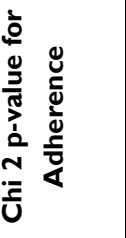 & $\begin{array}{l}\overline{8} \\
\text { ठ } \\
\mathrm{v}\end{array}$ & $\begin{array}{l}\bar{o} \\
0 \\
\text { v }\end{array}$ & $\begin{array}{l}\bar{\delta} \\
\text { ị } \\
\text { v }\end{array}$ & $\begin{array}{l}\bar{Q} \\
\grave{0} \\
\dot{0}\end{array}$ & $\begin{array}{l}\bar{o} \\
\bar{o} \\
\dot{0} \\
\dot{v} \\
\dot{v}\end{array}$ & 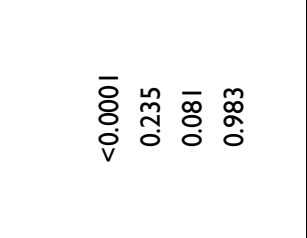 & 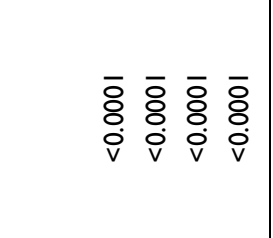 & $\begin{array}{l}\overline{8} \\
\text { ठ } \\
\text { v }\end{array}$ \\
\hline 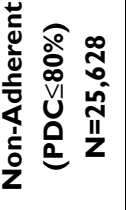 & i & 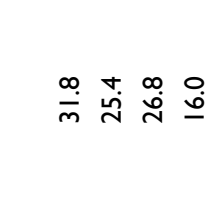 & $\stackrel{\infty}{\mathfrak{Y}}$ & 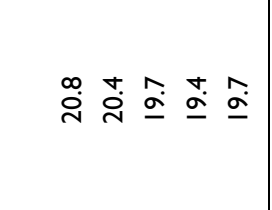 & $\hat{\vec{q}} \stackrel{+}{\Im}$ & 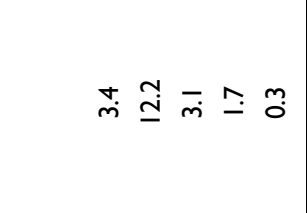 & 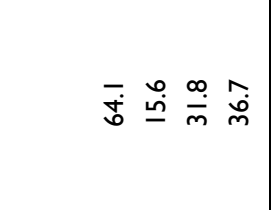 & $\circ \stackrel{\circ}{\mathrm{i}}$ \\
\hline 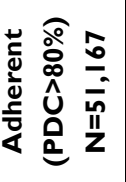 & 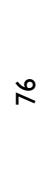 & 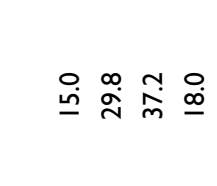 & $\hat{\dot{f}}$ & 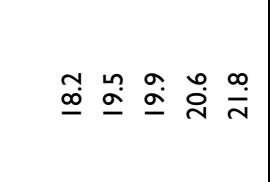 & $\stackrel{0}{\dot{H}} \stackrel{n}{\mathrm{i}}$ & 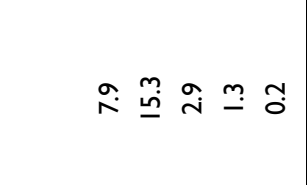 & 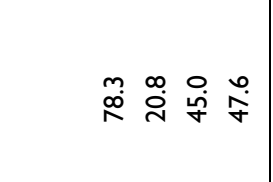 & 으임 \\
\hline 『̃ & $\stackrel{n}{\kappa}$ & 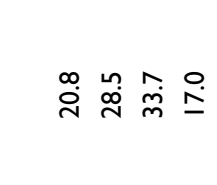 & $\stackrel{\circ}{\dot{j}}$ & 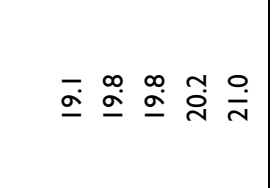 & $\frac{a}{i n} \bar{m}$ & 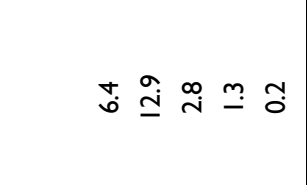 & 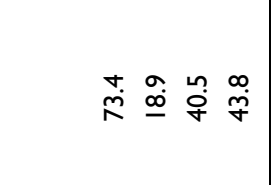 & $\stackrel{\dot{0}}{\circ}$ \\
\hline 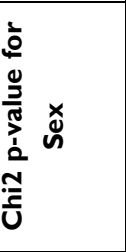 & $\begin{array}{l}\bar{Q} \\
\text { ठ̣. } \\
\mathrm{v}\end{array}$ & 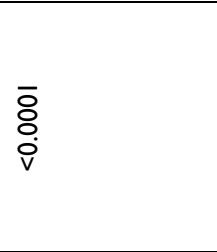 & 1 & $\begin{array}{l}\overline{8} \\
\text { ¿ } \\
\text { v }\end{array}$ & $\begin{array}{ll}\bar{\delta} & \bar{o} \\
0 & 0 \\
\dot{v} & \dot{0} \\
v\end{array}$ & 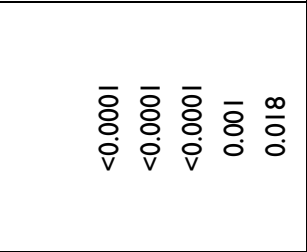 & 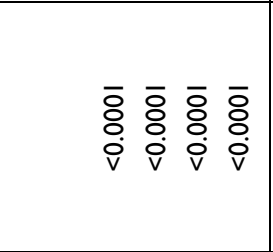 & $\begin{array}{l}\bar{\delta} \\
\text { ठ } \\
\circ \dot{0} \\
\mathrm{v}\end{array}$ \\
\hline 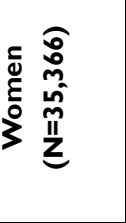 & $\stackrel{\infty}{\sim}$ & 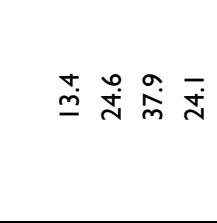 & 1 & $\stackrel{t}{\infty} \stackrel{a}{\sigma} \stackrel{m}{\stackrel{\alpha}{N}} \frac{a}{N}$ & $\underset{\dot{H}}{+\pi}$ & $\stackrel{0}{\sim} \stackrel{\sim}{\sim}=\stackrel{m}{0}$ & 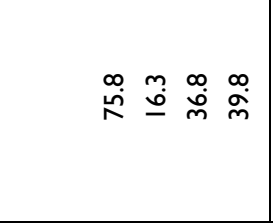 & $\stackrel{\infty}{\stackrel{\infty}{\infty}} \stackrel{\infty}{\sim}$ \\
\hline 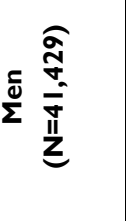 & $\pi$ & 공 & 1 & 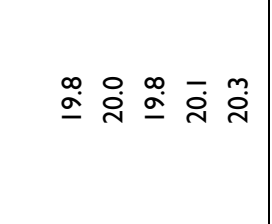 & 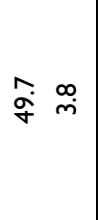 & $\stackrel{\infty}{\sim} \stackrel{m}{\underline{i}} \underset{m}{m} \stackrel{+}{=} \tilde{O}$ & 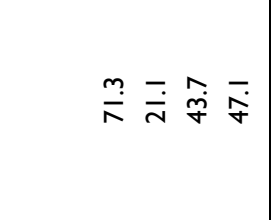 & fì \\
\hline & 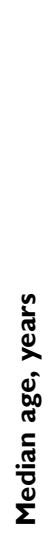 & 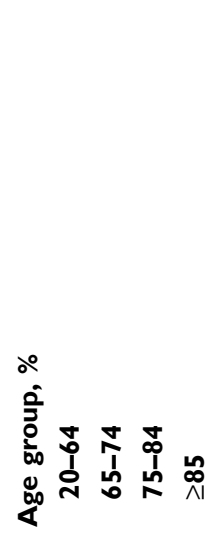 & 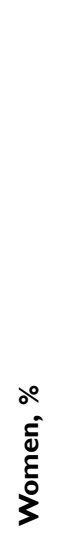 & 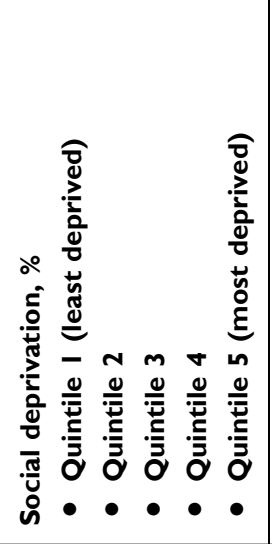 & 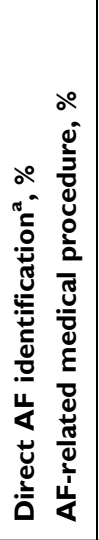 & 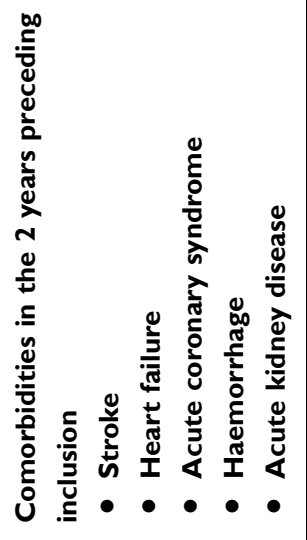 & 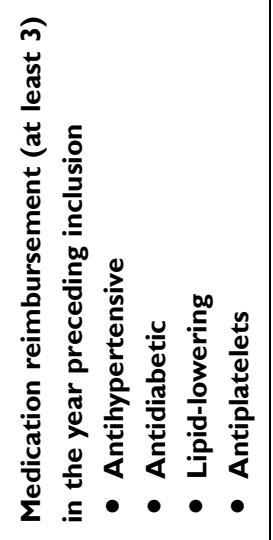 & 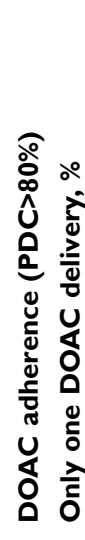 \\
\hline
\end{tabular}




\begin{tabular}{|c|c|c|}
\hline $\begin{array}{l}\bar{o} \\
\text { o. }\end{array}$ & $\begin{array}{llll}1 & 1 & 1 & 1\end{array}$ & 1 \\
\hline mà & 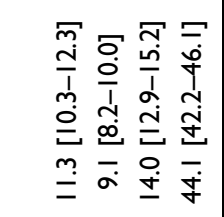 & ঃ \\
\hline $\bar{s} \stackrel{\circ}{\circ}$ & 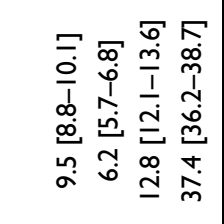 & ஓ \\
\hline ڤ̊ & 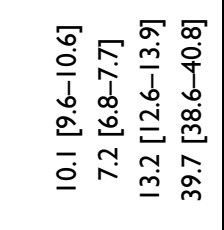 & ঃ \\
\hline $\begin{array}{l}\overline{8} \\
\text { ¿ } \\
\text { v }\end{array}$ & $\begin{array}{llll}1 & 1 & 1 & 1\end{array}$ & 1 \\
\hline 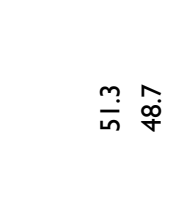 & 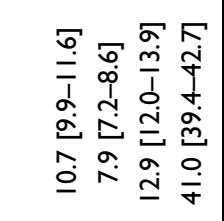 & ঃ \\
\hline 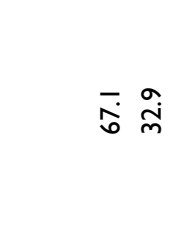 & 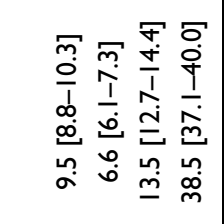 & 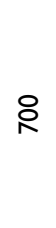 \\
\hline 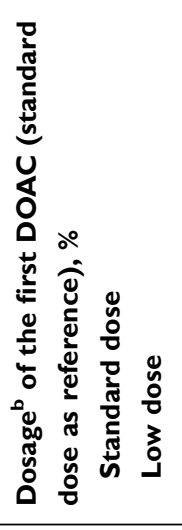 & 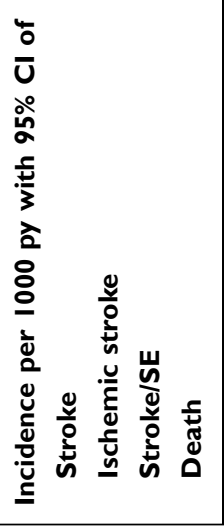 & 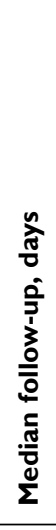 \\
\hline
\end{tabular}

1.04-1.17 for death) and those treated for diabetes (SHR 1.21, 95\% CI: 1.12-1.31, and CHR 1.40, 95\% CI: 1.35-1.45).

Hospitalization for acute coronary syndrome in the 2 years preceding inclusion was associated with an increased risk of stroke only (SHR 1.23, 95\% CI: 1.03-1.46), while heart failure and haemorrhages were associated with an increased risk of death only (CHR 2.09, 95\% CI: 2.01-2.18, and CHR 1.53, 95\% CI: 1.37-1.70, respectively). Furthermore, female sex was associated with a lower risk of death, and social deprivation with a higher risk of death but not of stroke.

\section{Discussion}

Our study showed a stroke incidence of 10.1 [9.6-10.6] per 1000 person-year in AF patients newly treated with DOAC in France in 2015 and a competitive risk of mortality of 39.7 [38.6-40.8] over a median follow-up of 23 months. Nonadherence to DOAC treatment was associated with an important increased risk of stroke and death. Female sex was associated with a lower risk of death but not of stroke. History of stroke and diabetes was strongly associated with increased risks of both stroke and death, whereas acute coronary syndrome was associated with an increased risk of stroke alone, and heart failure decompensation, social deprivation based on the area of residence, and haemorrhage were associated with an increased risk of death alone.

Non-adherence to DOAC treatment defined as $\mathrm{PDC} \leq 80 \%$ was associated with an increased risk of stroke and death by $42 \%$ ( $80 \%$ for ischemic strokes, see Supplementary Table II) and 38\%, respectively. A similar increased risk of Major Adverse Cardiovascular Events was related to non-adherence to DOAC (see Supplementary Table II). Sensitivity analyses using higher PDC showed the same results, and supplementary analyses in the group of patients with $\mathrm{PDC}>80 \%$ showed an increased risk of stroke with a $1 \%$ PDC decrease when introduced as a continuous variable in the model (data not shown). Furthermore, non-adherence to treatment had a similar negative impact in both low and high stroke risk patients (Supplementary Table III) and in all age groups (Supplementary Table IV). Very few studies have been published to date on the association between DOAC adherence and outcomes in AF patients. Yao and colleagues showed similar results in a large US administrative claims database compared to our study ${ }^{7}$ as well as clinical trials. ${ }^{12}$ Our study highlights the importance of addressing adherence issues in AF patients treated with DOAC. Moreover, we found an increased risk of stroke in patients treated with low-dose DOAC, as recently shown in the 


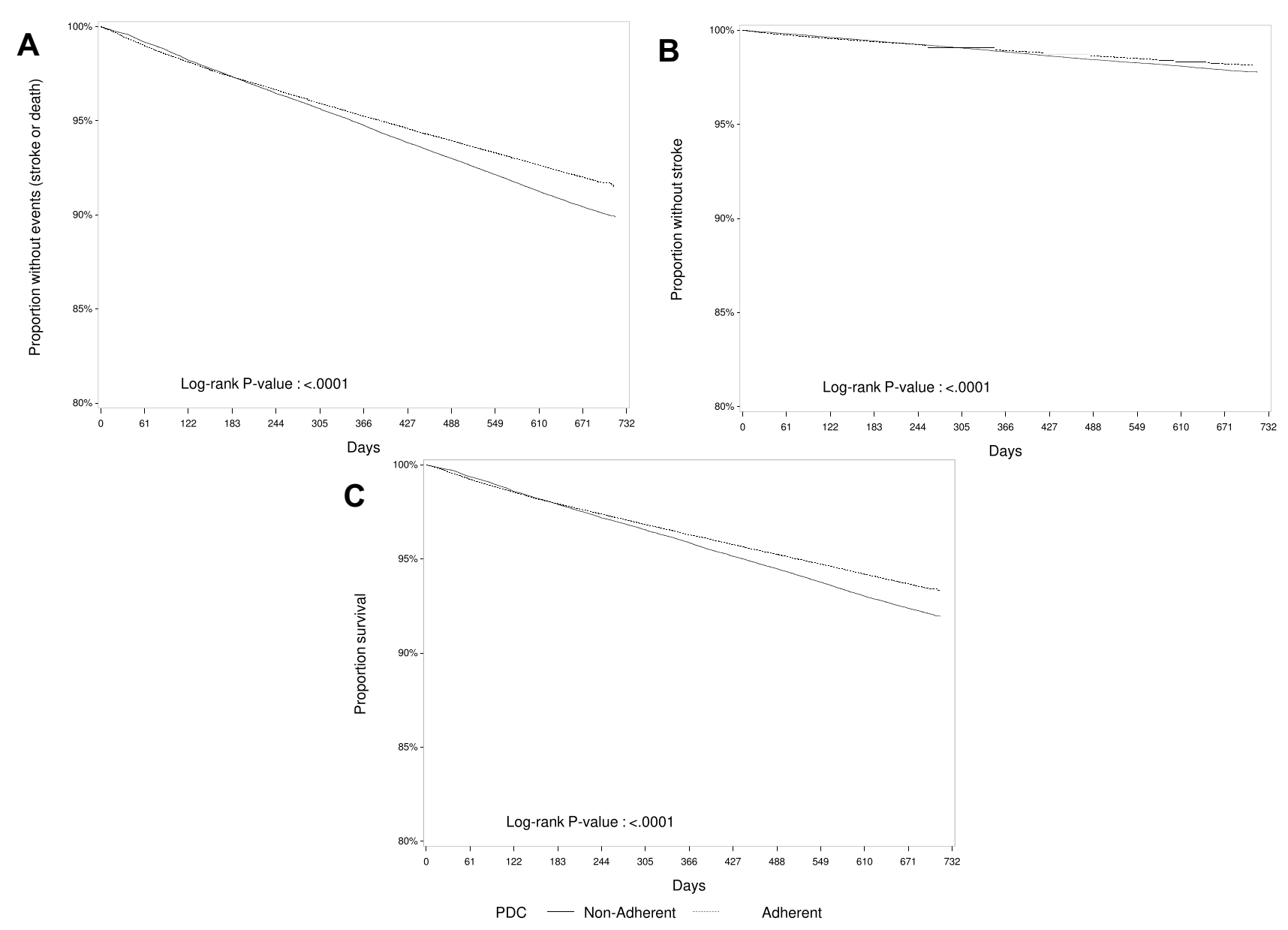

Figure I Kaplan-Meier curves for (A) all events of interest (stroke and death), (B) stroke, and (C) death. Abbreviation: PDC, percentage of days covered by direct oral anticoagulant treatment.

Italian RENo study. ${ }^{13}$ In our study, the proportion of adherence increased between 2012 (60\%, data not shown) and 2015 (67\%), concomitantly with a decrease in low-dose DOAC therapy at DOAC initiation, which is encouraging. However, patients were followed for almost 2 years, whereas DOAC therapy is a lifelong treatment; adherence impact should therefore be studied over a longer period.

Sex was not associated with stroke incidence after all adjustments in our study and in sensitivity analyses with either ischemic stroke or all strokes/SE as outcomes (see Supplementary Table II). Likewise, in PREFER-AF, sex was not mentioned as a stroke predictor in anticoagulated patients. ${ }^{14} \mathrm{~A}$ meta-analysis showed that women were at a higher risk of stroke in AF treated with VKA but not those treated with DOAC. ${ }^{15}$ Several recent studies including both anticoagulated and non-anticoagulated patients did not show an excess risk of stroke in women after adjustment. $^{16}$ The lower risk of death (all causes) in women observed in our study was also found in a previous study. ${ }^{17}$

A history of heart failure decompensation was strongly associated with death in our study, as shown previously in the literature among general AF patients, including in a few studies examining AF patients treated with DOAC. ${ }^{18}$ No significant association was found between heart failure and stroke in our study. Another study did not detect heart failure as an independent predictor of stroke in AF patients treated with OAC (VKA or DOAC), ${ }^{19}$ whereas PREFER-AF found heart failure to be an independent risk of stroke. No other trials or cohorts to date have investigated the role of heart failure in the AF population treated with OAC. Our finding is therefore interesting, as heart failure is not shown to be an independent risk factor of stroke in AF patients receiving DOAC. This could be explained by the fact that AF patients with heart failure are at a higher risk of stroke, because of left ventricular thrombus formation ${ }^{20}$ and 


\begin{tabular}{|c|c|c|c|c|c|c|c|c|c|c|c|c|}
\hline \multirow{3}{*}{ 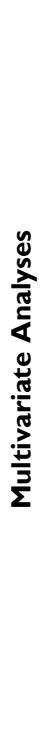 } & 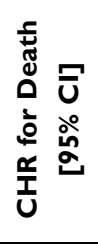 & 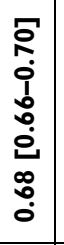 & & 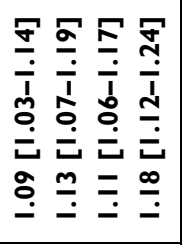 & 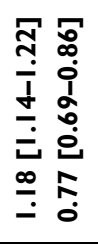 & & 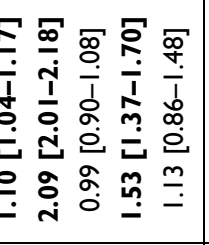 & & 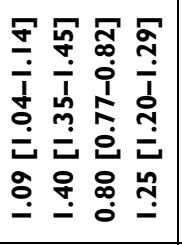 & 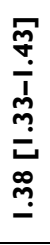 & 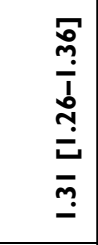 & 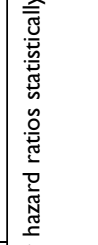 \\
\hline & 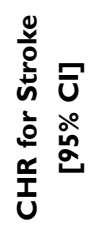 & 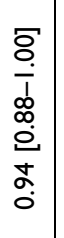 & & 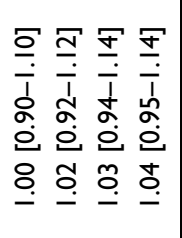 & 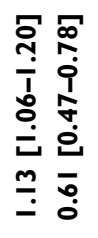 & & 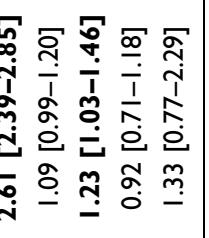 & & 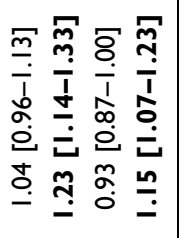 & 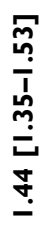 & 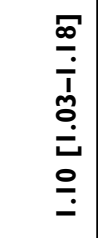 & $\begin{array}{c}\underline{5} \\
\grave{\lambda}\end{array}$ \\
\hline & 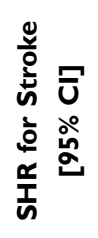 & $\begin{array}{l}\widetilde{c} \\
\dot{\delta} \\
\bar{\vdots} \\
\vdots \\
\dot{0} \\
\alpha \\
\alpha \\
0\end{array}$ & & 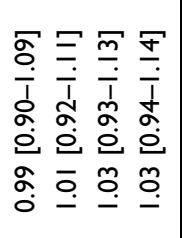 & 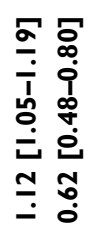 & & 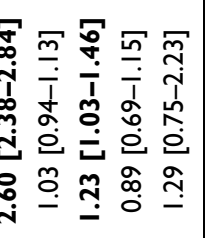 & & 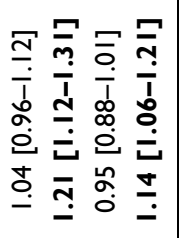 & 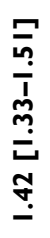 & 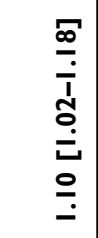 & 更 \\
\hline \multirow{3}{*}{. } & 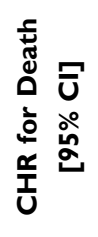 & 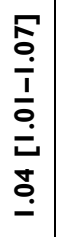 & & 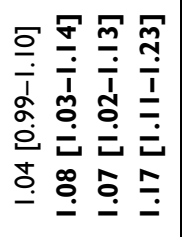 & 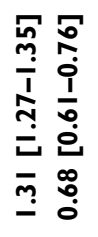 & & 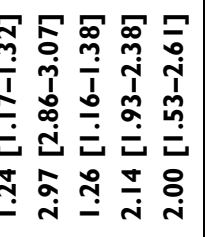 & & 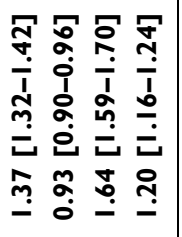 & 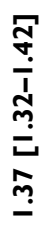 & 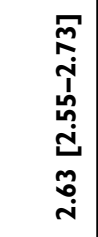 & $\vec{\Delta}$ \\
\hline & 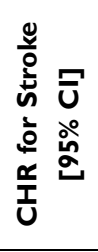 & 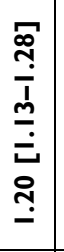 & & 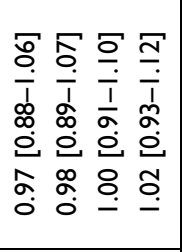 & 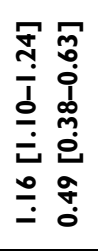 & & 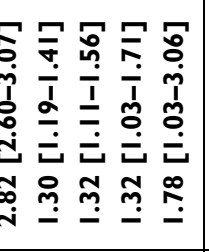 & & 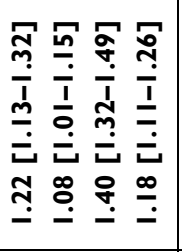 & 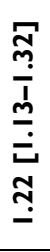 & 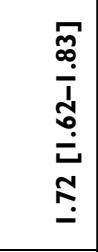 & 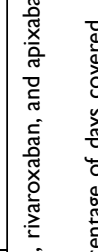 \\
\hline & 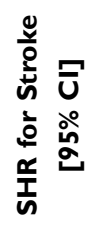 & 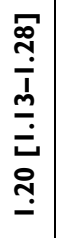 & & 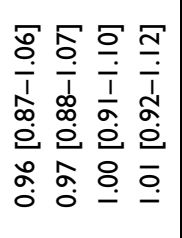 & 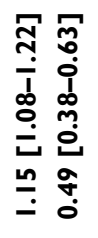 & & 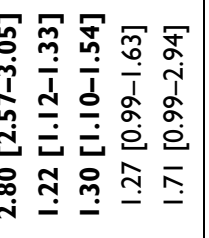 & & 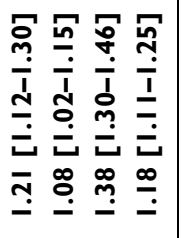 & 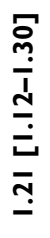 & 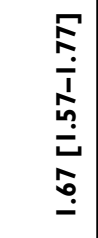 & 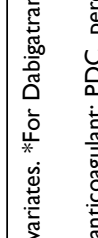 \\
\hline & & 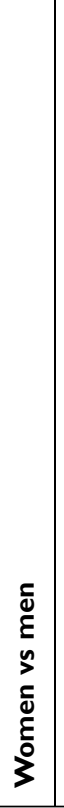 & 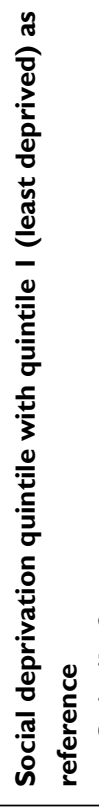 & 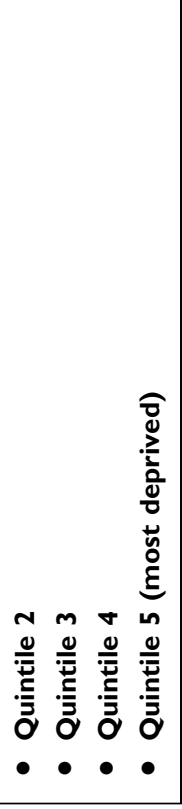 & 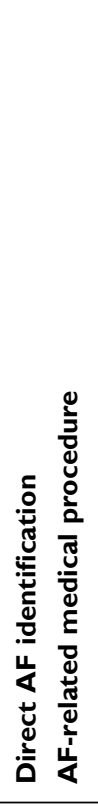 & 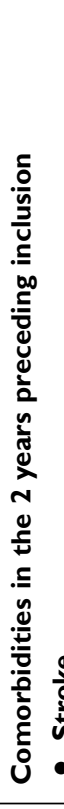 & 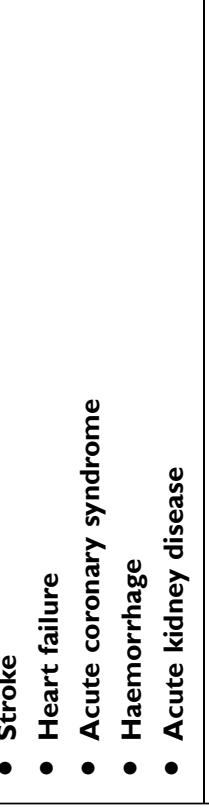 & 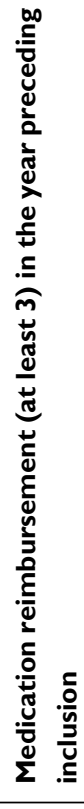 & 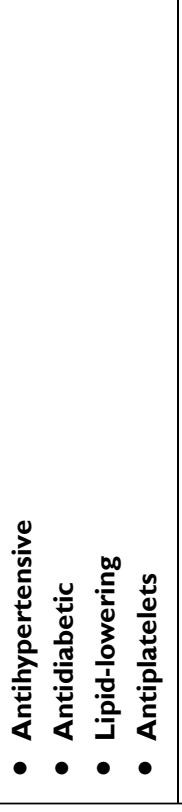 & 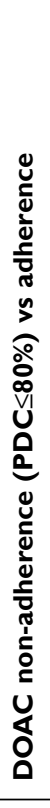 & 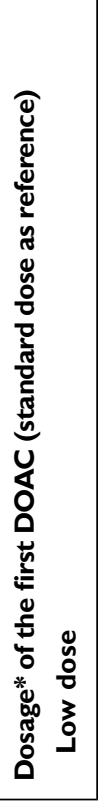 & 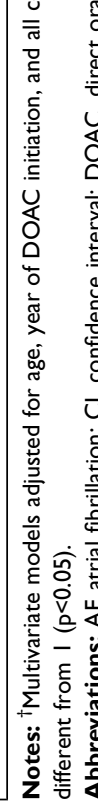 \\
\hline
\end{tabular}


general prothrombotic state, ${ }^{21}$ which might be prevented under DOAC. Evidence is nevertheless lacking, and the underlying mechanism remains unclear. However, we found that history of heart failure was associated with an increased risk of stroke in patients aged under 65 years (SHR 1.51, 95\% CI 1.12-2.03; Supplementary Table IV), which could be explained by the different pathogenesis of stroke in younger AF patients with heart failure.

Recent hospitalization for stroke, acute coronary syndrome, antiplatelet drugs, and diabetes treatment were all associated with a significantly higher risk of stroke in our study, thus highlighting the fact that these conditions remain independent risk factors of stroke in $\mathrm{AF}$ patients treated with $\mathrm{DOAC}$ as for general $\mathrm{AF}$ patients. $^{22}$

We found similar rates of stroke compared to registries of AF patients treated with DOAC in the US ${ }^{13,23,24}$ or the PREFER-AF study based on "real-world" AF patients treated with DOAC across Europe. ${ }^{14}$ However, lower rates of stroke were observed in a Japanese multicentre registry. ${ }^{25}$ Many studies provide outcome rates in AF patients, but they include both anticoagulated and non-anticoagulated $\mathrm{AF}$ patients (GARFIELD-AF, EORP-AF), ${ }^{18,26} \mathrm{AF}$ patients anticoagulated with DOAC and VKA, or hospitalized AF patients alone. ${ }^{27}$ Although the stroke risk is reduced in $\mathrm{AF}$ patients treated with DOAC compared to untreated patients, ${ }^{28} \mathrm{AF}$ still remains a risk factor for stroke and death. ${ }^{29}$

Although our study is based on a large population of unselected AF patients from all over France, several limitations should be noted. Firstly, a few risk factors and clinical characteristics such as tobacco smoking, obesity, and AF type were not available in the databases. Secondly, the reasons for non-adherence were not mentioned. However, according to a previous study, the majority of treatment discontinuation or non-adherence is due to patient decision/will and not because of a contraindication or new conditions leading to treatment termination. ${ }^{30}$ Regardless of the reasons, non-adherence to OAC therapy puts patients at a higher risk of stroke and death according to our results. Therefore, it is to carefully consider the indications for stopping DOAC treatment. We limited our study to AF patients treated with DOAC, as adherence to VKA drugs is difficult to determine using administrative claims databases, since these drugs are breakable. Finally, we did not have access to the mechanisms leading to stroke. Some risk factors could therefore be related to non-cardioembolic stroke and thus unrelated to AF. In the RENo study, $30 \%$ of strokes occurring in $\mathrm{AF}$ and treated with OAC were non-cardioembolic in origin. ${ }^{13}$ Finally, what we observed in France might not be generalized to other populations.

\section{Conclusion}

Among AF patients newly treated with DOAC, both stroke and death risks remain non-negligible. Several risk factors identified in AF patients were still associated with these outcomes in our population of AF patients treated with DOAC, whereas others like acute decompensation for heart failure were not independent stroke risk factors. We also showed an important excess risk of stroke and death in non-adherent patients. Adherence issues therefore need to be addressed, as they represent a challenge to the benefits of DOAC and, more generally, OAC in AF patients.

\section{Data Sharing Statement}

As a governmental agency, we had full access to the data and the right to publish the resulting study. Data cannot be shared as access to the SNDS data has to be approved by the French Data Protection Authority. Only French governmental agencies have access to SNDS for now.

\section{Ethics Approval}

As a health research institute, the French Public Health Agency has permanent access to the SNDS database approved by decree and by the French Data Protection Authority. According to French governmental regulations, the examination of the study protocol by the National Ethics Committee was not required.

\section{Consent to Participate}

Not applicable as patient and public were not involved in our research.

\section{Consent for Publication}

Not applicable as patient and public were not involved in our research.

\section{Author Contributions}

AG have drafted and written the article. AG, EC, CG, and VO contributed to the conception, study design, execution, acquisition of data, analysis and interpretation, and substantially revised and critically reviewed the article. AV, PT and YB contributed to the interpretation, substantially revised and critically reviewed the article. All authors have 
agreed on the journal to which article will be submitted and on the present submission. All authors have reviewed and agreed on all versions of the article, and agreed to take responsibility and be accountable for the contents of the article.

\section{Funding}

There is no funding to report.

\section{Disclosure}

Yannick Béjot received honoraria for lectures and consulting fees from AstraZeneca, Daiichi-Sankyo, BMS, Pfizer, Medtronic, Servier, MSD France, Amgen, and BoehringerIngelheim. The authors report no other conflicts of interest in this work.

\section{References}

1. Camm AJ, Accetta G, Ambrosio G, et al. Evolving antithrombotic treatment patterns for patients with newly diagnosed atrial fibrillation. Heart. 2017;103(4):307-314. doi:10.1136/heartjnl-2016309832

2. Hendriks JM, Gallagher C, Sanders P. Ensuring adherence to therapy with anticoagulation in patients with atrial fibrillation. Heart. 2017;103 (17):1308-1309. doi:10.1136/heartjnl-2017-311305

3. Ozaki AF, Choi AS, Le QT, et al. Real-world adherence and persistence to direct oral anticoagulants in patients with atrial fibrillation: a systematic review and meta-analysis. Circulation. 2020;13(3): e005969. doi:10.1161/CIRCOUTCOMES.119.005969

4. Liu C, Du X, Jiang C, et al. Long-term persistence with newly-initiated warfarin or non-VKA oral anticoagulant (NOAC) in patients with non-valvular atrial fibrillation: insights from the prospective China-AF registry. Med Sci Monit. 2019;25:2649-2657. doi:10.12659/MSM.915875

5. Maura G, Billionnet C, Alla F, Gagne JJ, Pariente A. Comparison of treatment persistence with dabigatran or rivaroxaban versus vitamin $\mathrm{K}$ antagonist oral anticoagulants in atrial fibrillation patients: a competing risk analysis in the french national health care databases. Pharmacotherapy. 2018;38(1):6-18. doi:10.1002/phar.2046

6. Hurtado-Navarro I, García-Sempere A, Rodríguez-Bernal C, SantaAna-Tellez Y, Peiró S, Sanfélix-Gimeno G. Estimating adherence based on prescription or dispensation information: impact on thresholds and outcomes. A real-world study with atrial fibrillation patients treated with oral anticoagulants in Spain. Front Pharmacol. 2018;9:1353. doi:10.3389/fphar.2018.01353

7. Yao X, Abraham NS, Alexander GC, et al. Effect of adherence to oral anticoagulants on risk of stroke and major bleeding among patients with atrial fibrillation. J Am Heart Assoc. 2016;5(2). doi:10.1161/ JAHA.115.003074.

8. Tuppin P, Rudant J, Constantinou P, et al. Value of a national administrative database to guide public decisions: from the systeme national d'information interregimes de l'Assurance Maladie (SNIIRAM) to the systeme national des donnees de sante (SNDS) in France. Revue d'epidemiologie et de sante publique. 2017;65(Suppl 4):S149-S167. doi:10.1016/j.respe.2017.05.004

9. Gabet A, Béjot Y, Olié V. Estimation of the incidence of atrial fibrillation treated by oral anticoagulants in France from 2010 to 2016. Arch Cardiovasc Dis Suppl. 2019;11(1):82. doi:10.1016/j.acvdsp.2018. 10.182
10. Rey G, Jougla E, Fouillet A, Hemon D. Ecological association between a deprivation index and mortality in France over the period 1997-2001: variations with spatial scale, degree of urbanicity, age, gender and cause of death. BMC Public Health. 2009;9:33. doi:10.1186/1471-2458-9-33

11. Fine JP, Gray RJ. A proportional hazards model for the subdistribution of a competing risk. J Amer Statist Assoc. 1999;94:496-509.

12. Sherwood MW, Douketis JD, Patel MR, et al. Outcomes of temporary interruption of rivaroxaban compared with warfarin in patients with nonvalvular atrial fibrillation: results from the rivaroxaban once daily, oral, direct factor Xa inhibition compared with vitamin $\mathrm{K}$ antagonism for prevention of stroke and embolism trial in atrial fibrillation (ROCKET AF). Circulation. 2014;129(18):1850-1859. doi:10.1161/CIRCULATIONAHA.113.005754

13. Paciaroni M, Agnelli G, Caso V, et al. Causes and risk factors of cerebral ischemic events in patients with atrial fibrillation treated with non-vitamin $\mathrm{K}$ antagonist oral anticoagulants for stroke prevention. Stroke. 2019:Strokeaha119025350.

14. Rohla M, Weiss TW, Pecen L, et al. Risk factors for thromboembolic and bleeding events in anticoagulated patients with atrial fibrillation: the prospective, multicentre observational PREvention oF thromboembolic events - European Registry in Atrial Fibrillation (PREFER in AF). BMJ Open. 2019;9(3):e022478. doi:10.1136/bmjopen-2018-022478

15. Pancholy SB, Sharma PS, Pancholy DS, Patel TM, Callans DJ, Marchlinski FE. Meta-analysis of gender differences in residual stroke risk and major bleeding in patients with nonvalvular atrial fibrillation treated with oral anticoagulants. Am J Cardiol. 2014;113 (3):485-490. doi:10.1016/j.amjcard.2013.10.035

16. Renoux C, Coulombe J, Suissa S. Revisiting sex differences in outcomes in non-valvular atrial fibrillation: a population-based cohort study. Eur Heart J. 2017;38(19):1473-1479. doi:10.1093/eurheartj/ ehw613

17. Piccini JP, Simon DN, Steinberg BA, et al. Differences in clinical and functional outcomes of atrial fibrillation in women and men: two-year results from the ORBIT-AF registry. JAMA Cardiol. 2016;1 (3):282-291. doi:10.1001/jamacardio.2016.0529

18. Bassand JP, Accetta G, Al Mahmeed W, et al. Risk factors for death, stroke, and bleeding in 28,628 patients from the GARFIELD-AF registry: rationale for comprehensive management of atrial fibrillation. PLoS One. 2018;13(1):e0191592. doi:10.1371/journal. pone. 0191592

19. Claxton JS, MacLehose RF, Lutsey PL, et al. A new model to predict ischemic stroke in patients with atrial fibrillation using warfarin or direct oral anticoagulants. Heart Rhythm. 2019;16(6):820-826. doi:10.1016/j.hrthm.2018.12.005

20. Pullicino PM, Halperin JL, Thompson JL. Stroke in patients with heart failure and reduced left ventricular ejection fraction. Neurology. 2000;54(2):288-294. doi:10.1212/WNL.54.2.288

21. de Peuter OR, Kok WE, Torp-Pedersen C, Buller HR, Kamphuisen PW. Systolic heart failure: a prothrombotic state. Semin Thromb Hemost. 2009;35(5):497-504. doi:10.1055/s-0029-1234145

22. Stroke Risk in Atrial Fibrillation Working Group. Independent predictors of stroke in patients with atrial fibrillation: a systematic review. Neurology. 2007;69(6):546-554. doi:10.1212/01. wnl.0000267275.68538.8d

23. Lip GYH, Keshishian A, Li X, et al. Effectiveness and safety of oral anticoagulants among nonvalvular atrial fibrillation patients. Stroke. 2018;49(12):2933-2944. doi:10.1161/STROKEAHA. 118.020232

24. Boriani G, Proietti M, Laroche C, et al. Contemporary stroke prevention strategies in 11096 European patients with atrial fibrillation: a report from the EURObservational Research Programme on Atrial Fibrillation (EORP-AF) Long-Term General Registry. Europace. 2018;20(5):747-757. 
25. Sakuma I, Uchiyama S, Atarashi $\mathrm{H}$, et al. Clinical risk factors of stroke and major bleeding in patients with non-valvular atrial fibrillation under rivaroxaban: the EXPAND Study sub-analysis. Heart Vessels. 2019.

26. Boriani G, Proietti M, Laroche C, et al. Association between antithrombotic treatment and outcomes at 1-year follow-up in patients with atrial fibrillation: the EORP-AF general long-term registry. Europace. 2019.

27. Healey JS, Oldgren J, Ezekowitz M, et al. Occurrence of death and stroke in patients in 47 countries 1 year after presenting with atrial fibrillation: a cohort study. Lancet. 2016;388(10050):1161-1169. doi:10.1016/S0140-6736(16)30968-0

28. Wicke FS, Schaller MA, Karymova K, Beyer M, Muller BS. Ischemic stroke risk estimation in patients without oral anticoagulation: an observational cohort study based on secondary data from Germany. BMC Cardiovasc Disord. 2019;19(1):94. doi:10.1186/ s12872-019-1074-7
29. Freedman B, Martinez C, Katholing A, Rietbrock S. Residual risk of stroke and death in anticoagulant-treated patients with atrial fibrillation. JAMA Cardiol. 2016;1(3):366-368. doi:10.1001/ jamacardio.2016.0393

30. Vrijens B, Heidbuchel H. Non-vitamin K antagonist oral anticoagulants: considerations on once- vs. twice-daily regimens and their potential impact on medication adherence. Europace. 2015;17 (4):514-523. doi:10.1093/europace/euu311

\section{Publish your work in this journal}

Clinical Epidemiology is an international, peer-reviewed, open access, online journal focusing on disease and drug epidemiology, identification of risk factors and screening procedures to develop optimal preventative initiatives and programs. Specific topics include: diagnosis, prognosis, treatment, screening, prevention, risk factor modification,

Submit your manuscript here: https://www.dovepress.com/clinical-epidemiology-journal systematic reviews, risk \& safety of medical interventions, epidemiology \& biostatistical methods, and evaluation of guidelines, translational medicine, health policies \& economic evaluations. The manuscript management system is completely online and includes a very quick and fair peer-review system, which is all easy to use. 